\title{
Observation of Raman Forward Scattering and Electron Acceleration in the Relativistic Regime
}

\author{
Astorre Modena, Zulfikar Najmudin, A. E. Dangor, C. E. Clayton, K. A. Marsh, \\ C. Joshi, Fellow, IEEE, Victor Malka, C. B. Darrow, and C. Danson
}

\begin{abstract}
Raman forward scattering (RFS) is observed in the interaction of a high intensity $\left(>10^{18} \mathrm{~W} / \mathrm{cm}^{2}\right)$ short pulse $(<1 \mathrm{ps})$ laser with an underdense plasma $\left(n_{e} \sim \mathbf{1 0}^{19} \mathbf{c m}^{-3}\right)$. Electrons are trapped and accelerated up to $44 \mathrm{MeV}$ by the highamplitude plasma wave produced by RFS. The laser spectrum is strongly modulated by the interaction, showing sidebands at the plasma frequency. Furthermore, as the quiver velocity of the electrons in the high electric field of the laser beam becomes relativistic, various effects are observed which can be attributed to the variation of electron mass with laser intensity.
\end{abstract}

\section{INTRODUCTION}

$\mathbf{P}$ ARTICLE acceleration by laser-generated large-amplitude relativistic plasma waves has attracted a great deal of interest since it was first proposed by Tajima and Dawson [1]. They described three basic ideas for generating such plasma waves: the laser wakefield accelerator (LWFA), the plasma beatwave accelerator (PBWA), and the Raman forward scattering (RFS) instability. In the past decade there have been numerous experimental studies of the PBWA [2]-[4]. However, only with the recent developments in intense shortpulse lasers [5] have the experimental studies of the LWFA [6] and the recently proposed self-modulated LWFA [7]--[9] become feasible.

The self-modulation of a pulse by RFS of long pulses was well known, owing to its implications in the preheating of targets in laser fusion experiments. Recently, it was observed that short pulses (pulse-length a few times the plasma period $T_{p}=2 \pi / \omega_{p}$, where $\omega_{p}$ is the plasma frequency) of high intensity would self-modulate and produce a coherent wake into which external electrons could be injected. This is the basis for what is now referred to as the self-modulated LWFA [7], [8]. In some cases the modulation can be so strong that the plasma wave can evolve to wavebreaking amplitudes. In this case, copious amounts of self-trapped electrons can be generated, and no wake is left behind [10].

Manuscript received January 5, 1996; revised February 16, 1996. This work was supported in part by grants from the EPSRC, the EU, and the U.S. Department of Energy.

A. Modena, Z. Najmudin, and A. E. Dangor are with the Blackett Laboratory, Imperial College, London SW7 2AZ, U.K. (e-mail: a.modena@ic.ac.uk).

C. E. Clayton, K. A. Marsh, and C. Joshi are with the University of California at Los Angeles, Los Angeles, CA 90024 USA.

V. Malka is with LULI, Ecole Polytechnique, Palaiseau, France.

C. B. Darrow is with the Lawrence Livermore National Laboratory, Livermore, CA 94550 USA.

C. Danson is with the Rutherford Appleton Laboratory, Didcot, U.K. Publisher Item Identifier S 0093-3813(96)03817-9.
The self-modulation can be the result of two effects. In the first case, the electron density fluctuations associated with the plasma wave lead to a variation in group velocity which causes the laser to bunch longitudinally. This bunching, or equivalently modulation, is at the plasma period. The intensity peaks are $\pi / 2$ out of phase with the initial density modulation and so the instability can grow [11]. This is an alternative explanation to one-dimensional (1-D) RFS. Second, the density fluctuations can act as negative and positive lenses and cause the laser pulse to bunch transversely [12]. This effect can be enhanced by relativistic self-focusing. The longitudinal and transverse bunching can occur simultaneously and lead to complicated intensity contours [10].

Note that in the first case, the modulation is due to a periodic redistribution of the laser energy longitudinally; whereas in the second, the modulation is due to a transverse redistribution of the laser energy. These two effects are interrelated, and one can think of the second effect as the whole beam analog of Raman side-scatter where the transverse wavelength can be taken as the inverse of the spot size.

We report here on an experiment to investigate RFS. Our observations of spectral cascading are indicative that longitudinal bunching is playing a crucial role in our experimental parameters [10]. Some of the results we present have been published in a previous paper [13]. In this paper we give a more detailed account of the experiment. Besides the observation of wavebreaking and acceleration of self-trapped plasma electrons, we show new results that are consistent with the relativistic behavior of the RFS instability in the case of a high-intensity short-pulse laser.

The paper is planned as follows. We review briefly RFS and its theoretical background in Section II. The experimental details and the results are then given in Sections III and IV. We conclude with a summary in Section V.

\section{RFS}

The RFS [14] instability has long been studied for its importance in inertial confinement fusion and plasma-based laser particle acceleration. Unlike Raman backward scattering [14], [15], RFS has been observed directly only in relatively few experiments [16], [17], [6], all at lower intensities. This is due to the much lower growth rate of RFS in underdense plasmas. Only with the high intensities available with shortpulse lasers is the gain large enough to drive plasma waves to an amplitude large enough to be observable and suitable for particle acceleration. 
The RFS instability grows from low-amplitude plasma-wave noise which can be due to a variety of sources. In shortpulse experiments, the main noise source is the plasma wave generated ponderomotively behind the leading edge of the laser. Erosion of the leading edge due to pump depletion by Raman side or backward scattering, which grow quickly in the early part of the pulse, can further enhance the plasma wave noise [18].

RFS is the decay of the strong electromagnetic pump wave, of frequency and wavenumber $\left(\omega_{o}, k_{o}\right)$, into a plasma wave of frequency and wavenumber $\left(\omega_{p}, k_{p}\right)$ and two forward propagating electromagnetic cascades at the Stokes $\left(w_{0}-\right.$ $\left.n \omega_{p}\right)$ and anti-Stokes $\left(\omega_{0}+n \omega_{p}\right)$ frequencies. Here $\omega_{p}=$ $\left(n_{e} e^{2} / \epsilon_{0} m\right)^{1 / 2}$ is the plasma frequency, and $n$ is a positive integer. The spatial and temporal interference of these sidebands with the laser produces an electromagnetic beat envelope of frequency $\omega_{p}$, propagating synchronously with the plasma wave. This electromagnetic beatwave exerts a resonant force on the plasma electrons. Hence, the original noise-level plasma wave is reinforced. As it grows, it further enhances scatter into the Raman sidebands, thus closing the feedback loop for the instability. In the RFS instability the plasma wave does not need a resonant laser pulse (as in the case of the LWF) as the laser bunches itself to provide a sort of series of micro-pulses matching the resonant condition.

Mori et al. [11] have recently derived the spatiotemporal behavior of RFS for any laser intensity. They calculated the spatiotemporal gain to be $G=e^{g} /(2 \pi g)^{1 / 2}$ where

$$
g=\left[a_{0} / \sqrt{2}\left(1+a_{0}^{2} / 2\right)\right]\left(\omega_{p} / \omega_{0}\right)^{2}\left(\omega_{0} / c\right) \sqrt{x \varphi}
$$

Here

$$
a_{o}=e A / m c^{2}=0.86 \times 10^{-9} \lambda(\mu \mathrm{m}) \sqrt{I_{0}\left(\mathrm{~W} / \mathrm{cm}^{2}\right)}
$$

is the normalized vector potential of the laser, $x$ is the distance traveled into the plasma, and $\varphi / c$ is the length of time that the assumed constant-intensity pulse has interacted with the plasma at position $x$. The $\left(1+a_{0}^{2} / 2\right)$ factor in the gain is the relativistic correction, and it is due to the dependence of the electron mass (and hence the plasma frequency) on the laser intensity.

Fig. 1 shows the gain $G$ of the instability as a function of the normalized vector potential for three values of the electron density, chosen to be in the range of the experiment reported here. Similarly, the values for $x$ (350 $\mu \mathrm{m}$ equal to the Rayleigh length) and $\varphi / c$ ( $800 \mathrm{fs}$ equal to the typical pulselength) have been fixed to correspond to the experimental parameters. Note that near $a_{o} \sim 1$, when the laser intensity becomes "relativistic" (i.e., when the quiver velocity of the electrons in the laser electric field approaches $c$ which is at $I_{o} \sim 10^{18} \mathrm{~W} / \mathrm{cm}^{2}$ for a laser wavelength of $\left.1 \mu \mathrm{m}\right)$, the gain shows a maximum. The reason for this is that the plasma frequency is inversely proportional to the square root of the electron mass. As the intensity increases, the quiver velocity of the electrons increases, the effective plasma frequency decreases, and so does the gain. Also illustrated in the figure is the strong dependence of the gain with plasma density-a threefold increase in density gives rise to an increase in $G$

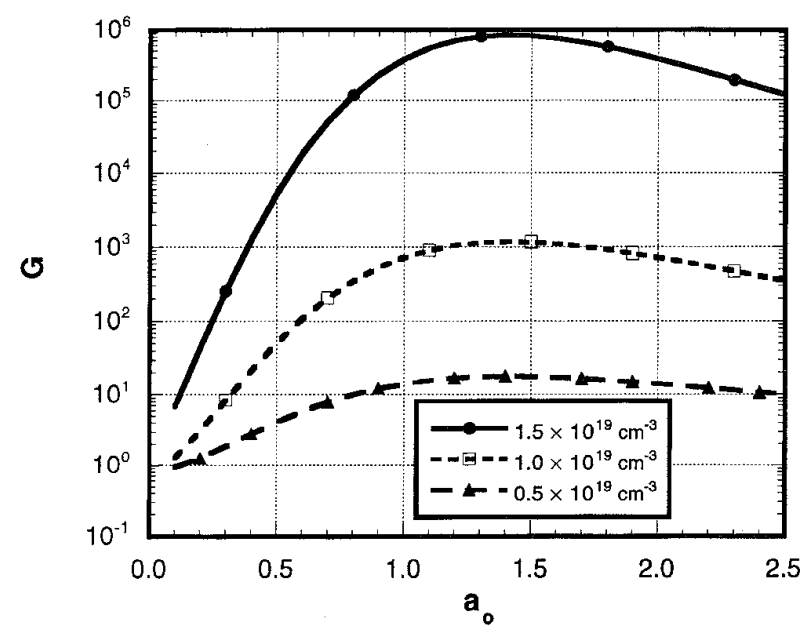

Fig. 1. RFS gain as a function of $a_{0}$ (laser intensity) at three plasma densities in the range of the experiment.

of many orders of magnitude. This is due to the exponential dependence of the gain.

\section{EXPERIMENTAL SETUP}

The experiment was conducted at the Central Laser Facility at the Rutherford Appleton Laboratory with the Vulcan [19] $\mathrm{Nd}$ :Glass $1.054 \mu \mathrm{m}$ laser system operating in the CPA configuration. The laser was able to provide $20 \mathrm{~J}$ in a $0.7-1 \mathrm{ps}$ pulse and was focused to a $20 \mu \mathrm{m}$ diameter spot. This corresponds to typical values of $20-25 \mathrm{TW}$ of power and intensities of the order of $5 \times 10^{18} \mathrm{~W} / \mathrm{cm}^{2}$.

A schematic of the experiment is shown in Fig. 2. The laser was focused by an $f / 5$ off-axis paraboloid mirror onto the welldefined front edge of a $4 \mathrm{~mm}$ diameter laminar plume of either helium or hydrogen gas. The plume was created by a pulsed supersonic gas jet [20] located $2 \mathrm{~mm}$ below the focal region. The typical laser intensity of $5 \times 10^{18} \mathrm{~W} / \mathrm{cm}^{2}$ is sufficient to fully ionize both helium and hydrogen over at least $2 \mathrm{~mm}$ into the jet. The plasma density, which varied with the backing pressure of the jet, was measured (through the frequency shift of the anti-Stokes sidebands) to be linear with backing pressure from 5 bar to at least 18 bar where the electron density was $1.5 \times 10^{19} \mathrm{~cm}^{3}$.

The two main diagnostics used were: 1) observation of the spectral distribution of the pump laser, forward scattered into an $f / 5$ cone corresponding to the pump laser beam, and 2) the energy spectrum of self-trapped electrons accelerated into an $f / 60$ cone centered on the laser axis. The forward scattered light, after passing through an attenuating pellicle and reflected by a beam steerer (both with small holes to allow unimpeded transmission of the accelerated electrons), was collected by a lens and dispersed in a spectrometer. The spectrum was detected with a liquid-nitrogen-cooled silicon 16-bit chargecoupled device (CCD) camera. With this arrangement, the dynamic range was sufficiently large (over $10^{5}$ ) to detect anti-Stokes sidebands up to the sixth. The dispersed spectral images were then processed by a computer and corrected for the spectral response of the detector and transmission curves 


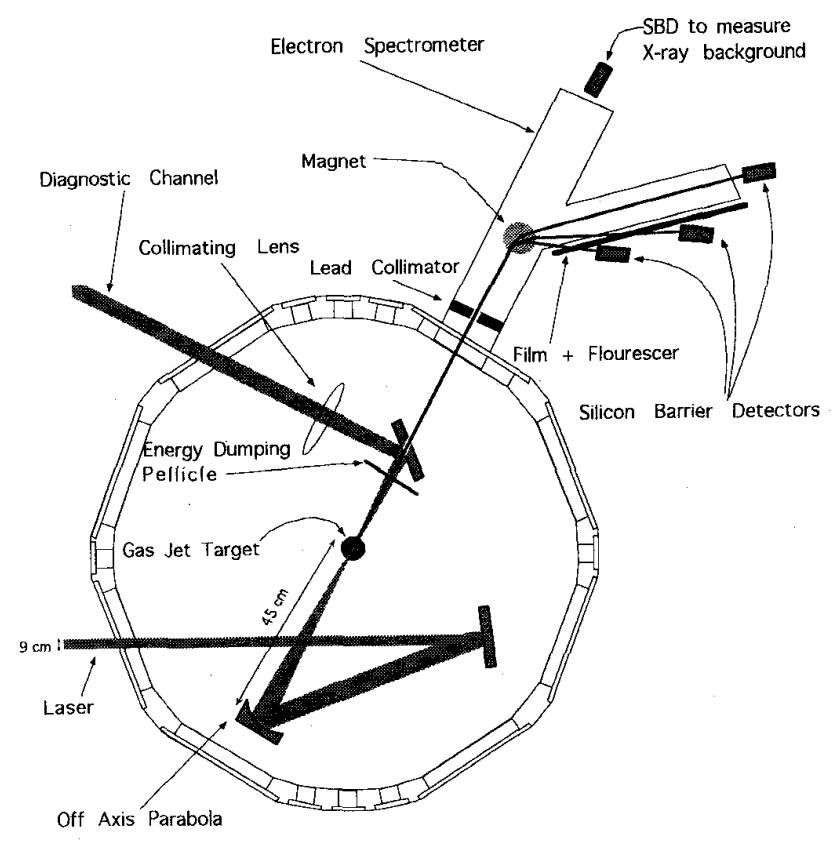

Fig. 2. Experimental setup.

of all the intervening optical components including any filters that were used. The sensitivity of the camera decreases rapidly for $\lambda>1.1 \mu \mathrm{m}$, so the Stokes sideband was only seen at low plasma density and not more than the first order was ever observed.

The electrons accelerated in the interaction passed through apertures drilled in the collecting optics and through a 4-cm thick lead collimator before entering the electron spectrometer. The spectrometer [21] was built in the Brown and Buechner design which covers a broad range of energies. The energy measurements by the magnetic spectrometer were limited to a maximum electron energy of $44 \mathrm{MeV}$ due to fieldsaturation of the electromagnet used. The vertical focusing of the spectrometer is weak, but the collection efficiency was not of importance due to the large number of accelerated electrons. The energy spectrum was measured in two ways. In the first method, the output of precalibrated biased silicon surface-barrier detectors was connected via preamplifiers to an oscilloscope to give the absolute number of electrons at discrete energies. A maximum of six detector channels were available, one of which was sometimes used to check X-ray levels at different locations. The second method utilized a fluorescer/film combination strip located (as with the silicon detectors) at the output plane of the spectrometer to give a continuous spectrum. Absolute numbers were obtained by cross calibration at a given energy with the signal from one silicon detector. The spectra, obtained with the two different methods, were in strikingly good agreement. A number of tests were made to check that we were measuring electrons instead of X-rays, and they all gave very convincing results [21].

The backscattered light at $1 \mu \mathrm{m}$ was also collected and spectrally resolved. Due to the high intensity of the laser, a huge amount of second harmonic was generated (conversion efficiency of $\sim 10^{-3}$ ). This second harmonic was measured

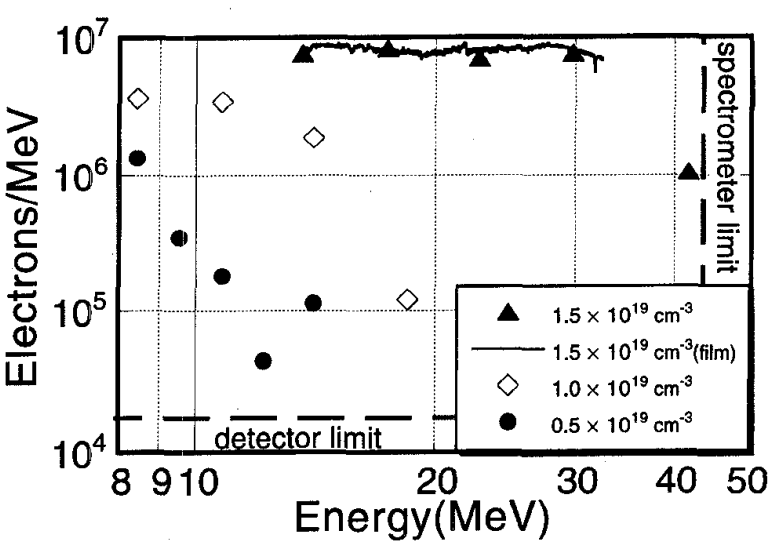

Fig. 3. Electron energy spectrum at three plasma densities in helium. All the single measurements were taken with the silicon detectors while the continuous curve was taken with the fluorescer/film combination.

both spectrally in the forward direction (which was also modulated by the plasma wave) and spatially at $30^{\circ}$, imaging it onto a CCD.

\section{RESULTS}

As mentioned above, the main diagnostics were the energy spectrum of the self-trapped accelerated electrons and the spectral distribution of the transmitted laser beam. We present these results first. We then present results which are directly attributable to relativistic effects associated with the intense laser intensities in this experiment. Finally, we present the backscatter results. The results obtained in helium and hydrogen do not show much difference at a given electron density. However, the observations obtained in helium were more extensive.

\section{A. Accelerated Electron Energy Spectra}

Fig. 3 shows the measured electron energy spectra in helium corresponding to the range of plasma density scanned (i.e., from 0.5 to $1.5 \times 10^{19} \mathrm{~cm}^{-3}$ ) [13]. All three cases were at a laser power of approximately $25 \mathrm{TW}$. The number of accelerated electrons at a given energy and the maximum electron energy both show a dramatic increase as the plasma density is increased to $1.5 \times 10^{19} \mathrm{~cm}^{-3}$. In particular, the number of electrons above $20 \mathrm{MeV}$ increases by at least three orders of magnitude. At the highest density, the accelerated electron distribution is rather flat up to $30 \mathrm{MeV}$, above which it begins to decrease with energy up to $44 \mathrm{MeV}$ which is the spectrometer limit. The flatness of the spectrum is due to the fact that the observation is made in only an $f / 60$ cone in the exact forward direction. The highest energy electrons are those that traverse the wave in the region of the highest longitudinal fields, i.e., right down the axis of the plasma wave. Lower energy electrons necessarily experience more of the radial fields and would tend to have a larger angular spread. Similar results were obtained in hydrogen as shown in Fig. 4. In this case, the number of higher energy electrons was lower and probably due to the fact that the flat spectrum behavior is not as prominent. 


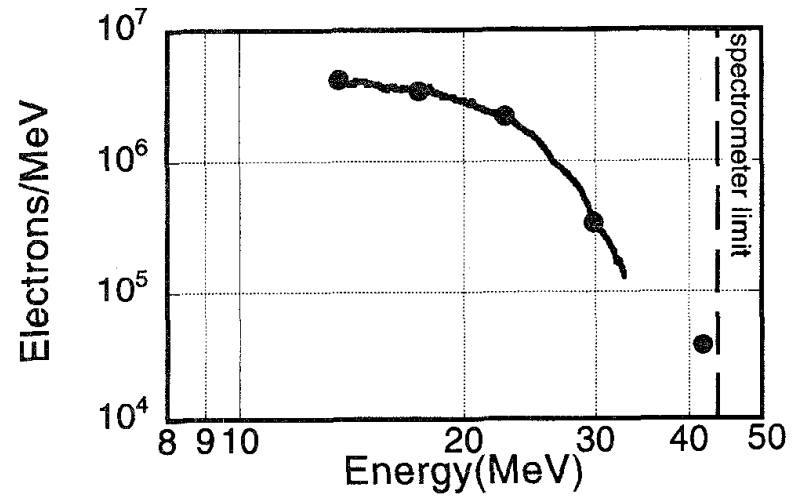

Fig. 4. Electron energy spectrum in hydrogen at $n_{e}=1.4 \times 10^{19} \mathrm{~cm}^{-3}$. All the single measurements were taken with the silicon detectors while the continuous curve was taken with the fiourescer/film combination.

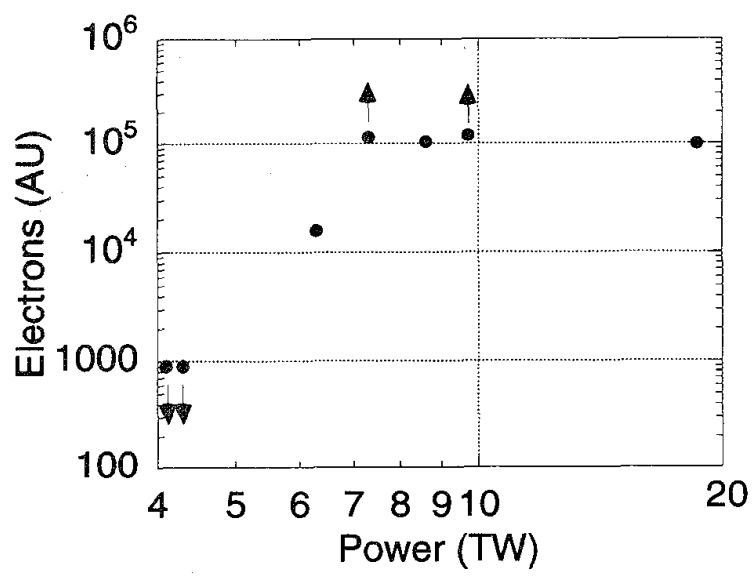

Fig. 5. Electron flux as a function of laser power. The downward arrows indicate the upper value of a measurement, while the upward arrows indicate the saturation of detectors and thus the minimum value of the measurements.

The major implication of these results is the huge electric field generated by the plasma wave. As dephasing limits the acceleration length to $\pi \gamma_{\phi}^{3} / k_{0} \approx 300 \mu \mathrm{m}$ (where $\gamma_{\phi}$ is the relativistic Lorentz factor for the phase velocity of the wave), the $44 \mathrm{MeV}$ that the electrons gain indicate a peak electric field of over $100 \mathrm{GV} / \mathrm{m}$. This represents the highest collective-wave field ever produced in a laboratory.

In Fig. 5 the flux of electrons accelerated to an energy of 11.5 MeV in hydrogen at a density of $8 \times 10^{18} \mathrm{~cm}^{-3}$, which is below the density at which wavebreaking occurred, is shown as a function of laser power. From the graph it is clear that above a certain power the number of accelerated electrons does not change significantly. This is in good agreement with the behavior of the theoretical RFS gain at high intensity plotted in Fig. 1. As explained above in Section II, the growth rate of RFS maximizes when the normalized vector potential $a_{0} \sim 1$ because of the relativistic increase in the electron mass. In Section IV-C we shall see that at the powers of 7-8 TW, where the flux of electrons appears to saturate, there are other indications that relativistic effects are taking place. This power corresponds to a focused intensity of $10^{18} \mathrm{~W} / \mathrm{cm}^{2}$ or

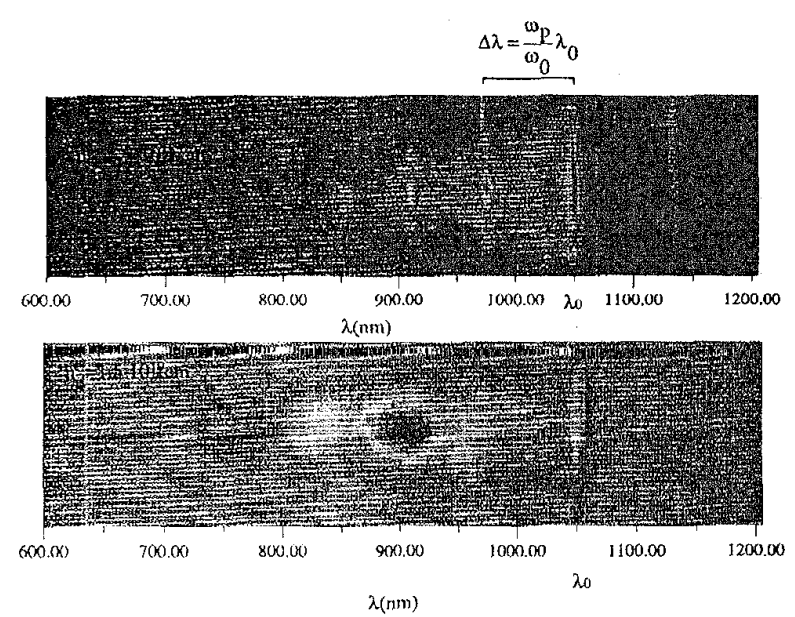

Fig. 6. Images of the laser spectrum after interaction with plasma.

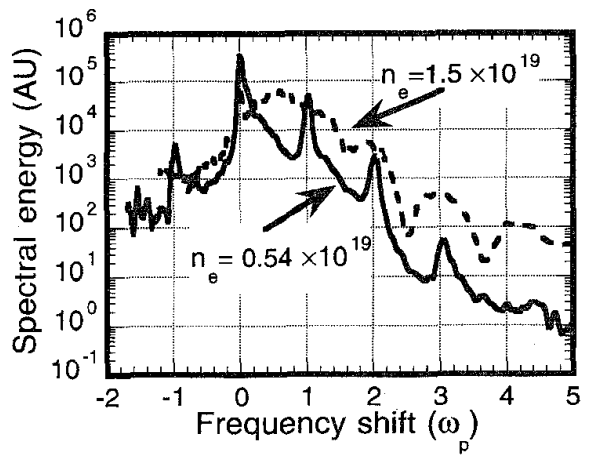

Fig. 7. Corrected laser spectra after proper renormalization.

$a_{0}=1$ which is in good agreement with the value of saturation described by the RFS theory.

\section{B. Transmitted Laser Spectra}

The photographs in Fig. 6 show the dispersed images of the transmitted laser light in helium at an electron density of 0.54 and $1.5 \times 10^{19} \mathrm{~cm}^{-3}$ obtained with the CCD camera at the output plane of the spectrometer. The images were processed, integrated vertically over space, and corrected for the transmission functions and sensitivity of both the optics and the instrument. They are replotted in Fig. 7 as a function of frequency to highlight the periodicity of the sidebands.

At the lower density, the upshifted anti-Stokes and downshifted Stokes signals at $\Delta \omega / \omega_{p}= \pm 1$ are clearly visible as is the unshifted laser and the second and third antiStokes sidebands. All the sidebands are sharply peaked. Their widths are very close to the laser bandwidth convoluted with the instrument function. (The value taken for the laser width is measured disregarding the shoulder due to ionization blueshift.) This indicates that the plasma is quite homogenous and that the plasma wave which generated these signals must have a coherence time on the order of the laser pulse. The dashed curve in Fig. 7 shows the spectrum when the plasma density is increased to $1.5 \times 10^{19} \mathrm{~cm}^{-3}$. Because the scale has 


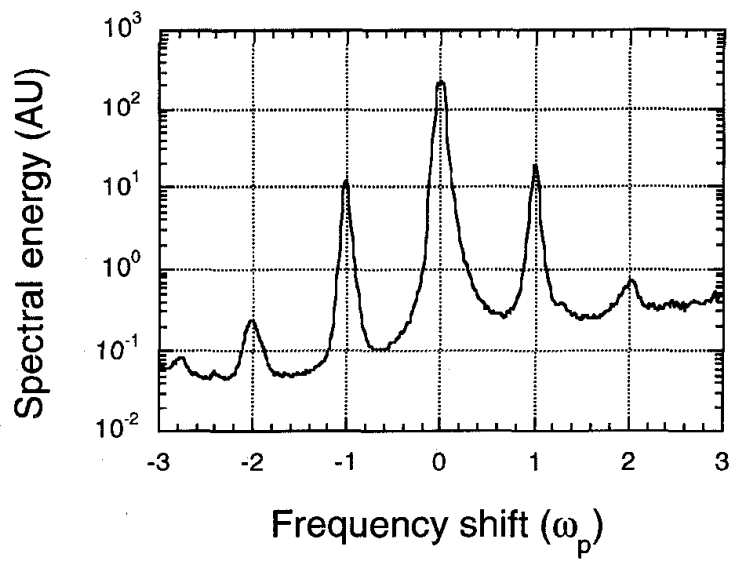

Fig. 8. Second harmonic spectrum after interaction with the plasma wave at $n_{e}=6 \times 10^{18} \mathrm{~cm}^{-3}$.

been normalized to $\omega_{p}$, the sidebands still have integer values on this scale. In this case the width of the sidebands is much larger and is of the order of the plasma frequency. This implies a coherence time of the order of only a few plasma periods.

The sharp increase in both the number and maximum energy of electrons as well as the sudden loss of coherence of the wave show that a strong distortion of the plasma wave has occurred, and we believe that this is characteristic of wavebreaking [13].

The creation and growth of the plasma wave was also monitored by its interaction with the second harmonic $(0.53$ $\mu \mathrm{m})$ light generated by the laser. This is equivalent to using a copropagating probe beam. Fig. 8 shows the second harmonic forward spectrum at a typical density of $6 \times 10^{18} \mathrm{~cm}^{-3}$. Various mechanisms may be creating the second harmonic light, the most probable of which is the interaction of the laser beam with plasma density gradients at the edges of the interaction area where nonfully ionized plasma is present. On top of the usual mechanisms, a new study [22] describes the possibility of second harmonic generation in homogenous plasmas due to relativistic effects which could suit the parameters in our experiment. Being a subject of interest in its own right, the generation of second harmonic will be the subject of another publication.

The spectrum of the second harmonic shows sidebands evenly spaced by $\omega_{p}$, indicating that the second harmonic is scattered by the plasma oscillations too. It is interesting to point out that all the plasma frequency harmonics are present, not only even ones, which indicates that the second harmonic of the laser was generated and then interacted with the plasma wave and not vice-versa. These measurements were also significant because they allowed us to have a complete spectrum with both Stokes and anti-Stokes sidebands without using a separate probe beam.

\section{Relativistic Effects}

At our laser intensities of more than $5 \times 10^{18} \mathrm{~W} / \mathrm{cm}^{2}$, the normalized laser vector potential $a_{0}$ can reach values higher than two. This means that taking a fully relativistic treatment

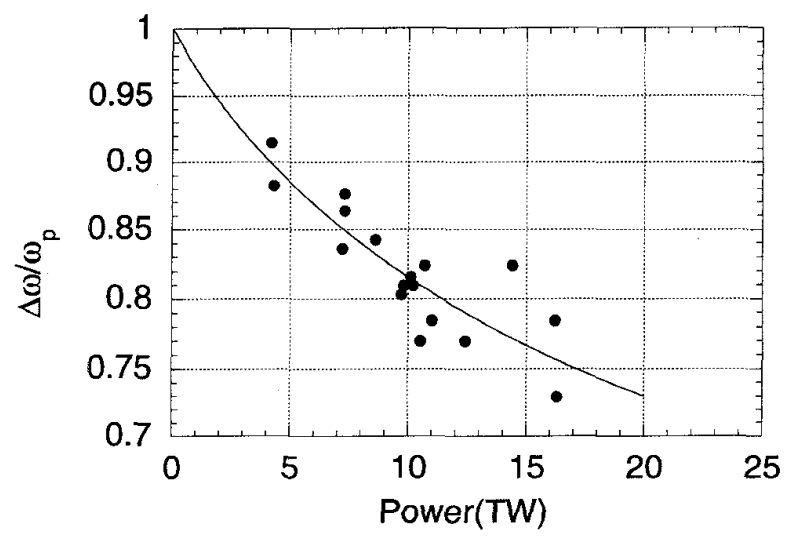

Fig. 9. Sideband frequency separation/plasma frequency as a function of laser power. The continuous curve is the fitted curve calculated with the formula $\omega_{p} / \omega_{p_{0}}=\left(1+a_{0}^{2} / 2\right)^{-(1 / 4)}$; the fit gives a $\omega_{p 0}=1.83 \times 10^{14} \mathrm{~s}^{-1}$ and a maximum intensity of $5 \times 10^{18} \mathrm{~W} / \mathrm{cm}^{2}$

is required to predict the behavior of the plasma. Since most theoretical treatments of such interactions have tended to treat $a_{0}$ as being small or at least smaller than one, these conditions are ripe for a host of anomalous or unpredicted behavior. We have already seen one such example in Fig. 5. This is the "saturation" effect that relativistic corrections have on the RFS growth and the corresponding saturation of the electron flux measured in the experiment.

A more impressive effect is the direct measurement of the change of electron mass with laser intensity. The electron mass change was observed directly through the separation of the sidebands in both the laser and the second harmonic spectra. As intensity increases, so the electron quiver velocity becomes relativistic. Thus the mass increases according to the relativistic Lorentz factor $\gamma=\left[1-\left(v_{\mathrm{osc}} / c\right)^{2}\right]^{-1 / 2}$ where $v_{\mathrm{osc}}$ is the oscillating velocity of the electrons in the laser field. As $\omega_{p}$ is inversely proportional to the electron mass, the actual separation of the sidebands should decrease with intensity. Of course a density depression due to a ponderomotive blowout may also contribute to a reduction in the shift. A simple treatment which considers only the space-charge forces and the laser ponderomotive force, and neglects ion motions, gives an estimate for the density depression of

$$
\frac{\delta n}{n}=\frac{1}{4 \pi^{2}} a_{0}^{2}\left(\frac{\lambda}{\sigma}\right)^{2}\left(\frac{\omega_{0}}{\omega_{p}}\right)^{2}
$$

where $\lambda$ is the laser wavelength and $\sigma$ is the focal spot diameter of the laser beam. Putting our initial parameters into this formula $\left(a_{0}=2, \sigma=20 \mu \mathrm{m}\right.$, and $\left.\omega_{0} / \omega_{p}=10\right)$, one finds that even at the highest intensity the shift would be not more than $3 \%$ which is quite small compared to the $40 \%$ change calculated due to the relativistic correction. These parameters do not consider relativistic self-focusing that can increase the laser intensity involved. In this case, though, the relativistic correction of the mass would increase as well, and the relative effect would not be so serious. So neglecting ponderomotive effects, we can calculate the estimated shift just on the basis of the relativistic change of the electron mass. 


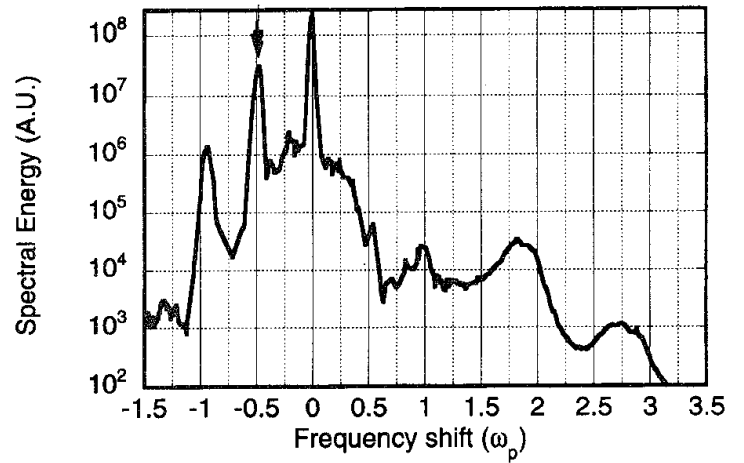

Fig. 10. Laser spectrum showing the presence of the $\omega-\omega_{p} / 2$ satellite (highlighted by the arrow).

This is plotted as the continuous curve in Fig. 9. The match between experimental results and calculation is remarkable. The same relativistic behavior has also been observed in two-dimensional (2-D) simulations using our experimental parameters [23], [24]. In the simulations as well, the effect appears to be mostly due to the relativistic quiver velocity. The sideband shows signs of a slight broadening with increasing laser power which can imply that the interaction occurs over a range of laser intensity regions.

Another sign that the interaction was in a highly relativistic regime is the observation of half harmonic creation in the laser spectrum. Fig. 10 shows a laser spectrum at a plasma density of around $10^{19} \mathrm{~cm}^{-3}$ in hydrogen. The Stokes sideband at frequency $\omega_{0}-\omega_{p} / 2$ is very clear and intense, though it seems to appear only at laser powers $P>10 \mathrm{TW}$. The result is erratic, but the power threshold is well established. This half harmonic of the forward Raman spectra has not been previously observed. However, it could be due to an instability recently proposed by Shvets et al.: the explosive Raman forward scattering instability [25] whose principal signature is the production of half-harmonic sidebands. The basic mechanism behind this instability is that at very high laser intensities, one must consider higher-order terms for the relativistic change in electron mass. The second-order term of the ponderomotive force $\left(\alpha\left(a^{2}\right)^{2}\right)$ introduces a term $\propto\left(a_{0} \cdot a_{1}\right)^{2}$, where $a_{1}$ is the scattered vector potential in the equation of motion of the electrons. This can be resonantly matched with plasma oscillations at frequency $\omega_{p} / 2$ thus allowing the half-harmonics to grow rapidly.

\section{Backscatter}

Backscattered spectra were also taken in helium. The results shown in Fig. 11 show similarities with the RFS spectra. At the low densities $\left(6 \times 10^{18} \mathrm{~cm}^{-3}\right)$, the spectrum is very sharply peaked at $\omega_{o}^{-} \omega_{p}$ with another peak at $\omega_{o}$; whereas, at the higher density the spectrum is highly modulated and very broad with far less energy in the frequency unshifted component at $\omega_{o}$. This unshifted signal is probably due to a combination of stimulated Brillouin scattering (SBS) [26] and a reflection due to dielectric discontinuity at the plasma jet boundary. At higher densities the Raman back, side, and forward scattering is so potent that the contribution of

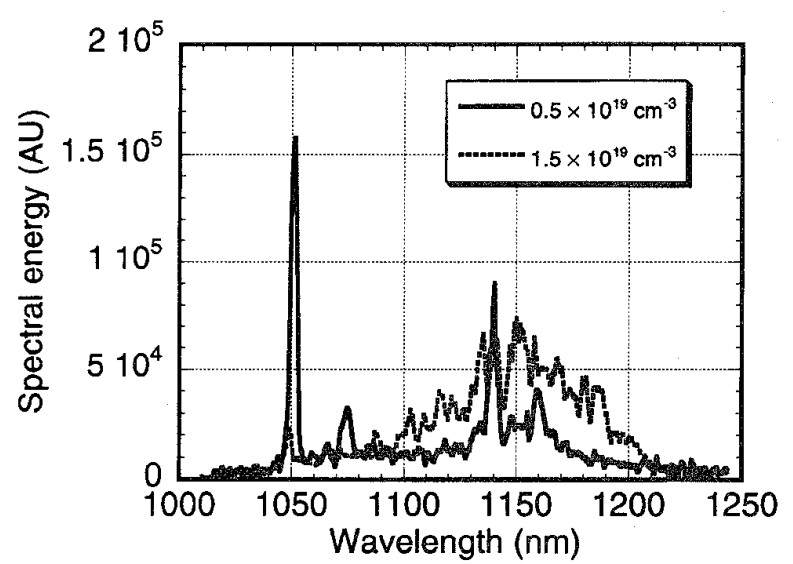

Fig. 11. Backscattered spectrum for two plasma densities. The drop of signal at around $1.2 \mu \mathrm{m}$ is due to the loss of sensitivity of the CCD camera.

the SBS to the backscatter near $\omega_{0}$ is reduced compared to lower densities. The exact cause of the very broad and highly modulated Raman backscatter is still unknown, but such features have been seen in other experiments and in simulations.

\section{CONCLUSION}

In summary, we have presented various results from the interaction of a high intensity laser beam with an underdense plasma. The mechanisms able to produce high-amplitude plasma waves were reviewed, and deeper background was given to RFS being the dominant phenomenon in our experimental regime. Initially we reviewed the results published previously in [13]. Among these results was the direct observation of RFS from the spectrum of the transmitted beam. This, in conjunction with the acceleration to high energy of trapped electrons, indicates the production of large-amplitude plasma waves. Also noted was the rapid broadening of the RFS signal above a certain density, allied with the rapid increase in the number of accelerated electrons, accelerated up to a record energy (from plasma-based devices) of $44 \mathrm{MeV}$. This behavior was attributed to the wavebreaking of plasma waves followed by the acceleration of the bulk of the plasma electron distribution.

Furthermore, we have presented new results which become apparent at relativistic laser intensities. The variation of the sideband separation with power and the observation of halfharmonic production may be explained invoking relativistic corrections to the electron mass. The former is simply due to the dependence on mass of the plasma frequency, while the latter relies on the second-order variation of the ponderomotive force due to the mass correction. Relativistic corrections are also summoned to explain the saturation of the RFS signal and the yield of accelerated electrons at the highest incident laser powers.

\section{ACKNOWLEDGMENT}

The authors wish to thank C. Stenz and R. Brückner of the Université d'Orléans for the design of the gas jet nozzle. 
Thanks are due also to W. B. Mori and G. Shvets for many insightful discussions and P. Chessa and K.-C. Tzeng for access to unpublished simulation results.

\section{REFERENCES}

[1] T. Tajima and J. Dawson, "Laser electron accelerator," Phys. Rev. Lett., vol. 43, p. 267, 1979.

[2] A. E. Dangor, A. K. L. Dymoke-Bradshaw, and A. E. Dyson, "Observation of relativistic plasma wave generated by the beat-wave with $1 \mu \mathrm{m}$ lasers," Physica Scripta, vol. 107, 1990.

[3] C. E. Clayton, K. A. Marsh, A. Dyson, M. Everett, A. Lal, W. P. Leemans, R. Williams, and C. Joshi, "Ultrahigh-gradient acceleration of injected electrons by laser-excited relativistic plasma waves," Phys. Rev. Lett., vol. 70, no. 37, 1993.

[4] F. Amiranoff, D. Bernard, B. Cros, F. Jacquet, G. Matthieussent, P. Mine, P. Mora, J. Morillo, F. Moulin, A. E. Specka, and C. Stenz, "Electron acceleration in Nd-laser plasma beat-wave experiments," Phys. Rev. Lett., vol. 74, pp. 5220-5223, 1995.

[5] M. D. Perry and G. Mourou, "Terawatt to petawatt subpicosecond lasers," Sci., vol. 264, pp. 917-924, 1994.

[6] K. Nakajima, D. Fisher, T. Kawakubo, H. Nakanishi, A. Ogata, Y. Kato, and Y. Kitagawa, "Observation of ultrahigh gradient electron acceleration by a self-modulated intense short laser pulse," Phys. Rev. Lett., vol. 74, pp. 4428-4431, 1995.

[7] J. Krall, A. Ting, E. Esarey, P. Sprangle, and G. Joyce, "Enhanced acceleration in a self-modulated laser wakefield accelerator," Phys. Rev. $E$, vol. 48, pp. 2157-2161, 1993; also "Propagation and guiding of intense laser-pulses in plasmas," Phys. Rev. Lett., vol. 69, p. 2200, 1992.

[8] N. E. Andreev, L. M. Gorbunov, V. I. Kirsanov, A. A. Pogosova, and R. R. Ramazashvili, "Self-resonant wake-field generation by intense lase pulse in plasmas," JETP Lett., vol. 55, p. 571, 1992.

[9] T. M. Antonsen and P. Mora, "Self-focusing and Raman-scattering of laser-pulses in tenuous plasmas," Phys. Rev. Lett., vol. 69, p. 2204, 1992.

[10] C. D. Decker, W. B. Mori, and T. Katsouleas, "Particle-in-cell simulations of Raman forward scattering from short-pulse high-intensity lasers," Phys. Rev. E, vol. 50, pp. R3338-R3341, 1994.

[11] W. B. Mori, C. D. Decker, D. E. Hinkel, and T. Katsouleas, "Raman forward scattering of short-pulse high-intensity lasers," Phys. Rev. Lett. vol. 72 , p. $1482,1994$.

[12] E. Esarey, J. Krall, and P. Sprangle, "Envelope analysis of intense laser pulse self-modulation in plasmas," Phys. Rev. Lett., vol. 73, pp. 3540-3543, 1994

[13] A Modena, A. E Dangor, Z Najmudin C. E Clayton, K Marsh C. Joshi, V. Malka, C. B. Darrow, C. Danson, D. Neely, and F. N. Walsh, "Electron acceleration from the breaking of relativistic plasma waves," Nature, vol. 377, pp. 606-608, 1995

[14] D. W. Forslund, J. M. Kindel, and E. L. Lindman, "Theory of stimulated scattering processes in laser-irradiated plasmas," Phys. Fluids, vol. 18, p. 1002,1975

[15] C. B. Darrow, C. Coverdale, M. D. Perry, W. B. Mori, C. Clayton, and K. Marsh, "Strongly coupled stimulated Raman backscatter from sub-picosecond laser," Phys. Rev. Lett., vol. 69, p. 442, 1992

[16] C. Joshi, T. Tajima, J. M. Dawson, H. A. Baldis, and N. A. Ebrahim, "Forward Raman instability and electron acceleration," Phys. Rev. Lett., vol. 47 , pp. $1285-1288,1981$

[17] C. A. Coverdale, C. B. Darrow, C. D. Decker, W. B Mori, K C Tzeng, K. A. Marsh, C. E. Clayton, and C. Joshi, "Propagation of intense subpicosecond laser-pulses through underdense plasmas," Phys. Rev. Lett., vol. 74, pp. 4659-4662, 1995.

[18] K.-C. Tzeng, W. B. Mori, and C. D. Decker, personal communication

[19] C. N. Danson, L. J. Barzanti, Z. Chang, A. E. Damerell, C. B. Edwards, S. Hancock, M. H. R. Hutchinson, M. H. Key, S. Luan, R. R. Mahadeo, I. P. Mercer, P. Norreys, D. A. Pepler, D. A. Rodkiss, I. N. Ross, M. A. Smith, R. A. Smith, P. Taday, W. T. Toner, K. W. M. Wigmore, T. B. Winstone, R. W. W. Wyatt, and F. Zhou, "High contrast multi-terawatt pulse generation using chirped pulse amplification on the VULCAN laser facility," Optics Commun., vol. 103, p. 392, 1993.

[20] R. Bruckner, "Etude de l'ionization laser de jets de gaz haute densite," Ph.D dissertation, Univ d'Orleans, 1994.

[21] C. E. Clayton, K. A. Marsh, C. Joshi, C. B. Darrow, A. E. Dangor, A. Modena, Z. Najmudin, and V. Malka, "A broadband electron spectrometer and electron detectors for laser accelerator experiments," in Proc. 1995 Particle Accelerator Conf., Dallas, TX, May 1-5, 1995.
[22] G. Shvets, "Interaction of intense lasers with plasmas," Ph.D. dissertation, MIT, 1995.

[23] P. Chessa, personal communication.

[24] K.-C. Tzeng and W. B. Mori, personal communication.

[25] G. Shvets, N. J. Fisch, and J.-M. Rax, "Relativistic Raman instability shifted by half-plasma frequency," Phys. Plasmas, to appear.

[26] D. Hinkel, E. A. Williams, and R. L. Berger, "Stimulated Brillouin backscattering of a short pulse laser," Phys. Plasmas, vol. 2, pp $3447-3460,1995$

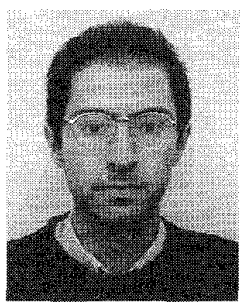

Astorre Modena was born in Italy in 1971. He received the first degree from the Hebrew University of Jerusalem and then went to Imperial College in London, where he is presently pursuing the Ph.D. degree in plasma physics.

His research interests include laser-plasma interactions with particular emphasis on laser acceleration schemes.

Zulfikar Najmudin was born in Uganda in 1970. He obtained the first degree in physics from Oxford University, Oxford, after which he completed the $\mathrm{Ph} . \mathrm{D}$. degree at Imperial College, London.

He is currently a Research Assistant in the Plasma Physics Group at Imperial College.

A. E. Dangor, photograph and biography not available at the time of publication.

C. E. Clayton, photograph and biography not available at the time of publication.

K. A. Marsh, photograph and biography not available at the time of publication.

C. Joshi (M'83-SM'88-F'93), photograph and biography not available at the time of publication.

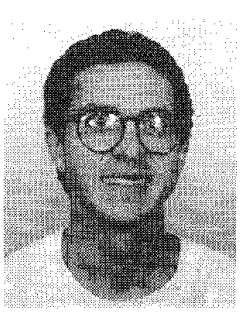

Victor Malka was born in 1960. He obtained the $\mathrm{Ph} . \mathrm{D}$. degree in plasma physics.

$\mathrm{He}$ is an established Researcher in the Laboratoire pour l'Utilisation des Lasers Intenses at Ecole Polytechnique, France. He has worked on implosionrelated experiments and is currently working in shor and long pulse laser interaction with underdense plasmas for both wakefield and inertial confinement fusion schemes.

C. B. Darrow, photograph and biography not available at the time of publication.

C. Danson, photograph and biography not available at the time of publication. 